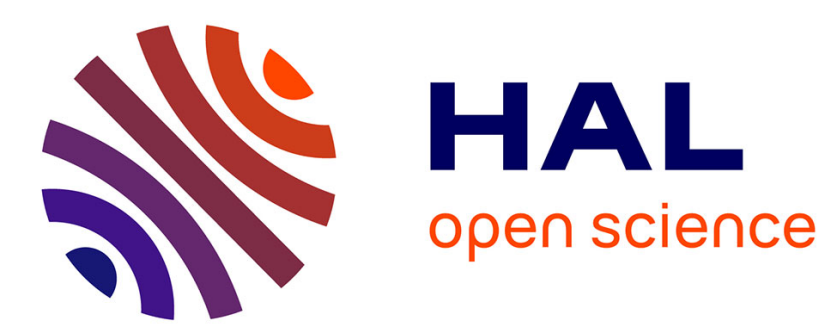

\title{
Maneuver Planning for Autonomous Vehicles, with Clothoid Tentacles for Local Trajectory Planning
}

\author{
Alia Chebly, Reine Talj, Ali Charara
}

\section{To cite this version:}

Alia Chebly, Reine Talj, Ali Charara. Maneuver Planning for Autonomous Vehicles, with Clothoid Tentacles for Local Trajectory Planning. 20th IEEE International Conference on Intelligent Transportation Systems (ITSC 2017), Oct 2017, Yokohama, Japan. pp.1-6, 10.1109/ITSC.2017.8317856 . hal-01648402

\section{HAL Id: hal-01648402 https://hal.science/hal-01648402}

Submitted on 25 Nov 2017

HAL is a multi-disciplinary open access archive for the deposit and dissemination of scientific research documents, whether they are published or not. The documents may come from teaching and research institutions in France or abroad, or from public or private research centers.
L'archive ouverte pluridisciplinaire HAL, est destinée au dépôt et à la diffusion de documents scientifiques de niveau recherche, publiés ou non, émanant des établissements d'enseignement et de recherche français ou étrangers, des laboratoires publics ou privés. 


\title{
Maneuver Planning for Autonomous Vehicles, with Clothoid Tentacles for Local Trajectory Planning
}

\author{
Chebly Alia and Talj Reine and Charara Ali \\ Sorbonne universités, Université de technologie de Compiègne \\ CNRS, Heudiasyc UMR 7253, CS 60319 \\ 60203 Compiègne, France \\ alia.chebly@hds.utc.fr reine.talj@hds.utc.fr ali.charara@hds.utc.fr
}

\begin{abstract}
Autonomous navigation is nowadays a very important topic not only in the research field, but also in the industry, academic and military fields. Indeed, to accomplish this autonomy, three essential modules are needed: The perception of the environment and the vehicle localization in it, the trajectory planning and the vehicle's control. The work presented in this paper covers the trajectory planning module and can be considered as an extension of previous works presented in [1], where we developed an algorithm for local trajectory planning based on Clothoid Tentacles method. In [1], the tentacles method is used to overtake a static obstacle and turn back to the reference trajectory defined by the right lane of the road. In this paper, we aim to ameliorate the tentacles method by studying the overtaking maneuver, and generating a suitable trajectory for the lane changing maneuvers taking into account the vehicle dynamics, the road rules and some security measurements. This trajectory will serve as an intermediate reference trajectory for the vehicle in the next few seconds. The local planning algorithm is then executed with the aim of tracking the generated trajectory while avoiding possible obstacles. The maneuver planning level added to the tentacles method in this work aims to simplify the planning task and to guarantee the vehicle stability and security.

Index Terms-Autonomous vehicles, maneuver planning, Overtaking, local planning, tentacles method, clothoids
\end{abstract}

\section{INTRODUCTION}

Trajectory planning is a key component in the autonomous navigation field. Indeed, the trajectory planning can be divided into three main levels: Route planning, Maneuver planning and local planning. The route planning level aims to generate the best global route from the given origin to the destination using real time traffic information. The second level, the maneuver planning, acts as the vehicle brain and tries to navigate in a human-like driving manner. The interaction with other traffic participants is interpreted at this level and the best maneuver to be executed is then chosen regarding the situation. Once the best maneuver is selected, the local planning is activated in order to find the best trajectory. At this level, the vehicle motion constraints, the comfort of the passenger, the smoothness of the trip and eventually the motion safety are considered. In this paper, the maneuver planning level, namely the overtaking maneuver as well as the local planning one are discussed. Several strategies were developed to seek with a safe overtaking maneuver. In [2], the optimal lane-change trajectory is obtained by minimizing the total kinetic energy, superimposing an optimally smooth, minimum-jerk trajectory upon the path. However, for the sake of simplicity and generality, the model does not explicitly take into account the dynamics of the vehicle or the vehicle model. This approach was used in [11], where an $H_{\infty}$ optimal controller is used to track the generated trajectory. In [9], [10], results are obtained for the distance to begin the diversion and the total time the lane-change maneuver takes, considering the vehicle dynamics. However, the trajectories they generate are not necessarily smooth and they do not obtain closedform formulas. Furthermore, they only consider lane-change maneuvers and not overtaking a moving vehicle. In [3], [13], the proposed algorithm determines whether there exists a longitudinal trajectory which allows the ego vehicle to safely position itself in a given gap on the target lane. If such a trajectory exists, the algorithm plans the corresponding lateral trajectory. The lane change trajectory planning problem is thereby reduced to solving low-complexity model predictive control problems resulting in loosely coupled longitudinal and lateral motion trajectories. Although this algorithm is promising, the computational time remains important. In [4], a fifth order polynomial trajectory is used to execute the lane changing maneuver. They assume that the ego vehicle and the preceding vehicle will not change their speeds during the overall overtaking maneuver. To take into account the vehicle dynamics and the obstacles avoidance, they proceed by adding a sixth degree with a sixth coefficient to the longitudinal equation of motion. Having only five boundary constraints, this added degree is set in order to avoid obstacles represented by s-topes forms and to guarantee the dynamic constraints. This method requires a pre-processing step to set the sixth degree before starting the lane change maneuver. Support vector machine [5], Bayesian Network [6], Fuzzy Logics [7] and many other approaches dealing with the overtaking maneuvers problem can be found in the literature. However, this kind of approaches needs a training phase and can be inefficient in evasive environment. In [12], a polynomial trajectory is generated to execute the lane changing, but the trajectory feasibility is post-treated using a simple vehicle model. If the trajectory is not feasible, they simply discard it and search another trajectory.

In this work, the overtaking maneuver is executed by gener- 
ating a polynomial trajectory taking into account the vehicle dynamics constraint as well as the traffic rules related to speed and safety constraints. The algorithm permits to generate the trajectory in a single step reducing the complexity as well as the computational time. At the local level, the tentacles method developed in [1] is used. Note that some improvements were conducted to the method cited in [1], such as the consideration of moving obstacles. In fact, the clothoids method, when used alone, does not allow the addition of the constraints that take into account the road rules and the recommended security distances. The added maneuver planning level permits a best manipulation of this decision step. The paper is organized as follows: Section II presents the planner architecture. The overtaking maneuver is then presented in Section III and the local planning method is presented briefly in Section IV. The simulation results are presented in Section V while Section VI concludes the paper.

\section{ARCHITECTURE}

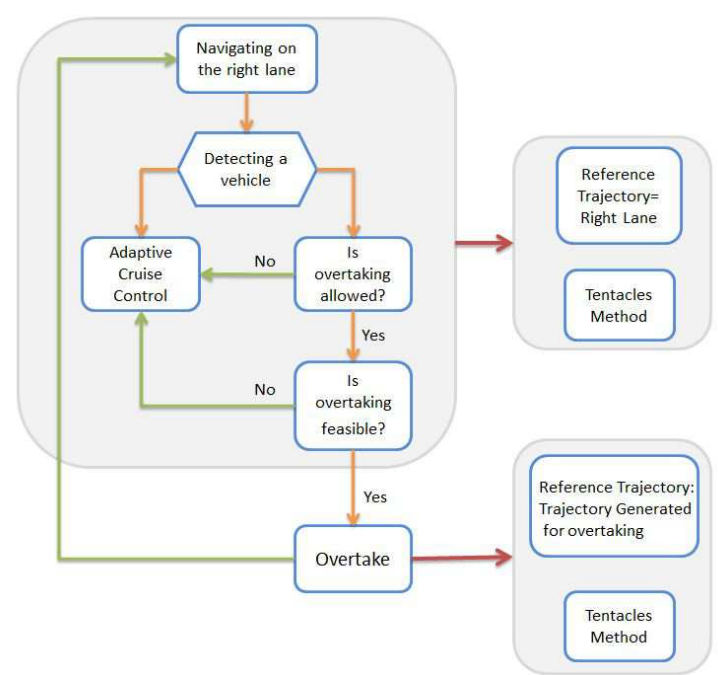

Fig. 1. Planning Architecture.

The navigation strategy is shown in Fig. 1. The vehicle starts navigating on the right lane. Once it detects a vehicle in front, the ego vehicle adapts its speed in order to maintain a security inter-vehicle distance corresponding to two seconds as defined by the road rules. (If the distance between the ego vehicle and the preceding vehicle becomes lower than two times the ego vehicle speed, the ego vehicle speed is adjusted to be $V_{x}=$ dist $_{o b s} / 2$.) While navigating on the right lane, this latter is considered as the reference trajectory and the tentacles method is executed in order to track this reference and avoid any unexpected collision.

The ego vehicle can overtake the preceding vehicle if and only if its desired speed is greater than the preceding vehicle speed by more than $20 \mathrm{~km} / \mathrm{h}$, and if the left lane is not occupied. For simplicity, we consider for the moment that the left lane is free. Once the vehicle decides to overtake, we check if there is any feasible trajectory that enables the lane changing without violating the dynamic constraints neither the security and the road rules. In fact, we consider that when the ego vehicle reaches the left lane center at a position $p$, the overtaken vehicle is at a position $p+m_{s}$ on the right lane, where $m_{s}$ is an additional security marge. Moreover, the lane change should be executed without exceeding the acceptable accelerations limits in order to guarantee the vehicle stability. If a suitable trajectory is found, the vehicle considers this trajectory as the new reference to track using the tentacles method. In other words, the tentacles method is now executed in order to track the generated trajectory for the lane changing maneuver while avoiding any possible collision. Once arrived to the left lane, the ego vehicle navigates on this lane until it can turn back to the right lane. Actually, this lane changing from the left to the right lane begins when the ego vehicle is positioned in front of the overtaken vehicle. In this maneuver, we should guarantee a safety distance of two seconds between the considered vehicles, once the ego vehicle reaches the right lane.

\section{MANEUVER Generation}

The overtaking maneuver is executed in three phases: Lane changing maneuver, navigating on the left lane and returning to the right lane. In the following, the vehicle A refers to the ego vehicle while the vehicle $B$ refers to the overtaken vehicle.

\section{A. Phase 1: Lane Changing maneuver}

In order to execute the lane changing maneuver, two polynomials trajectory are defined in the lateral and the longitudinal directions. The polynomials degrees are defined regarding the boundary conditions: The initial values of position, speed, and acceleration and two target values (at a certain time $T_{1}$ ) of speed and acceleration are set in the longitudinal direction while the initial and target values of position, speed, and acceleration are specified in the lateral direction. This defines a fourth-order and a fifth-order polynomial time description for longitudinal and lateral positions, respectively. The origin of the coordinate axis is fixed in the center of gravity of the vehicle, $\mathrm{x}$ being the longitudinal axis in the driving direction of the vehicle, and $y$ being the right-hand side perpendicular to $\mathrm{x}$. The equations of motion in the longitudinal and the lateral directions with respect to the time $t$ can be written as follows:

$$
\begin{aligned}
& x(t)=a_{0}+a_{1} t+a_{2} t^{2}+a_{3} t^{3}+a_{4} t^{4} \\
& y(t)=b_{0}+b_{1} t+b_{2} t^{2}+b_{3} t^{3}+b_{4} t^{4}+b_{5} t^{5}
\end{aligned}
$$

The boundary conditions are given by :

$$
\begin{aligned}
& x(0)=0, \dot{x}(0)=V_{A_{0}}, \ddot{x}(0)=0, \dot{x}\left(T_{1}\right)=V_{A_{f}}, \ddot{x}\left(T_{1}\right)=0 \\
& y(0)=0, \dot{y}(0)=\ddot{y}(0)=0, y\left(T_{1}\right)=w, \dot{y}\left(T_{1}\right)=\ddot{y}\left(T_{1}\right)=0
\end{aligned}
$$


After some computations, one can find the coefficients $a_{i}$ and $b_{j}$ of the equations in (1) as:

$$
\begin{aligned}
& a_{0}=0, a_{1}=V_{A_{i}}, a_{2}=0 \\
& a_{3}=\frac{V_{A_{f}}-V_{A_{i}}}{T_{1}^{2}}, a_{4}=\frac{V_{A_{i}}-V_{A_{f}}}{2 T_{1}^{3}} \\
& b_{0}=0, b_{1}=0, b_{2}=0 \\
& b_{3}=\frac{10 w}{T_{1}^{3}}, b_{4}=\frac{-15 w}{T_{1}^{4}}, b_{5}=\frac{6 w}{T_{1}^{5}}
\end{aligned}
$$

where $\mathrm{w}$ refers to the lane width, $T_{1}$ the lane change duration and $V_{A_{i}}$ and $V_{A_{f}}$ are the initial and the target speeds of the vehicle. These parameters $\left(T_{1}, V_{A_{i}}\right.$ and $\left.V_{A_{f}}\right)$ are set in such a way to take into account the vehicle dynamic constraints, the road rules dealing with the overtaking maneuver and the safety constraints.

The vehicle dynamic constraints are satisfied by limiting the longitudinal and the lateral accelerations of the trajectory $(\mathrm{x}, \mathrm{y})$. Let $a_{x_{\min }}$ and $a_{x_{\max }}$ be the boundary limits of the longitudinal acceleration and $a_{y_{\min }}$ and $a_{y_{\max }}$ be the boundary limits of the lateral acceleration. The vehicle accelerations profiles while changing the lane are represented by $\ddot{x}$ and $\ddot{y}$ given by:

$$
\begin{aligned}
& \ddot{x}(t)=2 a_{2}+6 a_{3} t+12 a_{4} t^{2} \\
& \ddot{y}(t)=2 b_{2}+6 b_{3} t+12 b_{4} t^{2}+20 b_{5} t^{3}
\end{aligned}
$$

The study of these profiles provides us with the extremum of these two functions:

The longitudinal acceleration has only one extremum at $t=$ $T_{1} / 2$, which corresponds to a maximum if $V_{A_{i}}<V_{A_{f}}$ and a minimum otherwise. We consider that we are not going to decelerate while changing the lane and we set $\ddot{x}\left(t=T_{1} / 2\right)<$ $a_{x_{\max }}$ leading to a constraint on the time duration of the lane changing maneuver:

$$
T_{1}>\frac{V_{A_{f}}-V_{A_{i}}}{2 / 3 a_{x_{\max }}} .
$$

The lateral acceleration presents a maximum at $t=t_{1}=$ $T_{1} / 2-0.29 T_{1}$ and a minimum at $t=t_{2}=T_{1} / 2+0.29 T_{1}$. Setting $\ddot{y}\left(t_{1}\right)<a_{y_{\max }}$ and $\ddot{y}\left(t_{2}\right)>a_{y_{\min }}$ leads to another constraint on the time duration of the lane changing maneuver:

$$
T_{1}>\max \left(\sqrt{\frac{5.77 w}{a_{y_{\max }}}}, \sqrt{\frac{-5.77 w}{a_{y_{\min }}}}\right) .
$$

In order to respect the road rules, the target speed $V_{A_{f}}$ should be greater than the overtaken vehicle speed by at least $20 \mathrm{~km} / \mathrm{h}$, while respecting the maximum speed allowed on the left lane:

$$
V_{A_{f}}=\min \left(\max \left(V_{A_{i}}, V_{B}+20 \mathrm{~km} / \mathrm{h}\right), V_{x_{\max }}^{L_{2}}\right),
$$

where $V_{B}$ is the overtaken vehicle speed and $V_{x_{\max }}^{L_{2}}$ is the maximum speed allowed on the left lane.

For safety reasons, we add another constraint that allows the ego vehicle to reach the left lane at a position slightly behind the position of the overtaken vehicle on the right lane (see Fig. 2).
To do so, we have to guarantee that :

$$
D_{1} \leq D_{o b s_{1}}+V_{B} T_{1}-m_{s},
$$

where $D_{1}$ is the longitudinal distance traveled by the ego vehicle during the lane changing maneuver, $D_{o b s_{1}}$ the distance between the ego vehicle and the overtaken vehicle at $t=0$ (at the beginning of the lane changing maneuver) and $m_{s}$ is the target distance between the projection of the vehicle $\mathrm{A}$ on the right lane and the position of the vehicle $\mathrm{B}$ on that lane at $t=T_{1}$.

Replacing $D_{1}$ by $x\left(t=T_{1}\right)=\left(V_{A_{f}}+V_{A_{i}}\right) T_{1} / 2$, and noting that $\left(V_{A_{f}}+V_{A_{i}}\right)>2 V_{B}$, Eq. (8) can be written as:

$$
T_{1} \leq \frac{2\left(D_{o b s_{1}}-m_{s}\right)}{V_{A_{f}}+V_{A_{i}}-2 V_{B}} .
$$

As a result, we define $V_{A_{f}}$ using (7), then we have to find $T_{1}$ that satisfies (5), (6) and (9), which means find $T_{1}$ that satisfies:

$$
\max \left(T_{\min 1}, T_{\min 2}\right) \leq T_{1} \leq T_{\max }
$$

with:

$$
\begin{aligned}
T_{\min 1} & =\max \left(\sqrt{\frac{5.77 w}{a_{y_{\max }}}}, \sqrt{\frac{-5.77 w}{a_{y_{\min }}}}\right) \\
T_{\min 2} & =\frac{V_{A_{f}}-V_{A_{i}}}{2 / 3 a_{x_{\max }}}, \\
T_{\max } & =\frac{2\left(D_{o b s_{1}}-m_{s}\right)}{V_{A_{f}}+V_{A_{i}}-2 V_{B}} .
\end{aligned}
$$

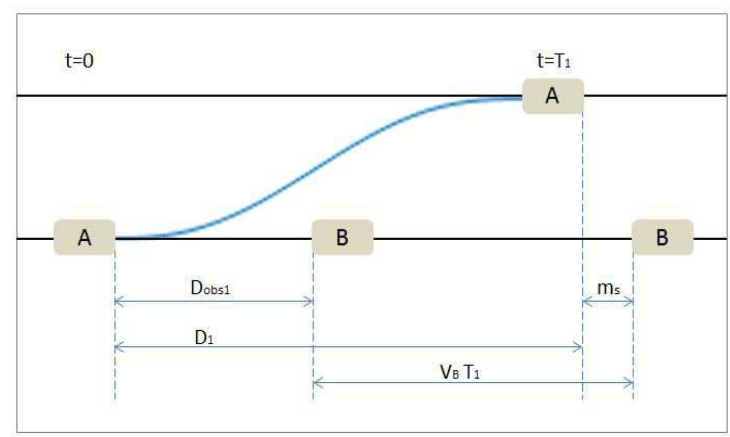

Fig. 2. Phase 1: Lane changing maneuver.

If a solution for Eq. (10) exists (e.g. $\max \left(T_{\min 1}, T_{\min 2}\right)<$ $T_{\max }$ ), we set $T=T_{\max }$ in order to guarantee the equality of the constraint (9). If we can not find a suitable value for $T_{1}$, (e.g. $\left.\max \left(T_{\min 1}, T_{\min 2}\right)>T_{\max }\right)$, we claim that the lane changing maneuver can not be safely executed without violating the dynamic constraints of the vehicle.

\section{B. Phase 2: Navigating on the left lane}

For simplicity, we assume that, during this phase, both of the vehicles $\mathrm{A}$ and $\mathrm{B}$ maintain their velocities $V_{A_{f}}$ and $V_{B}$, respectively. Assuming that the vehicle A will begin the returning phase when it passes the vehicle B by a certain safety margin $m_{s 2}$, then the traveling distance on the left lane $\left(D_{2}\right)$ 
can be computed in function of the traveling time on the left lane $\left(T_{2}\right)$ as (see Fig. 3):

$$
\begin{aligned}
D_{2} & =m_{s}+m_{s 2}+L_{A}+L_{B}+V_{B} T_{2} \\
& =V_{A_{f}} T_{2}
\end{aligned}
$$

The traveling time on the left lane is then given by:

$$
T_{2}=\frac{m_{s 2}+m_{s}+L_{A}+L_{B}}{V_{A_{f}}-V_{B}},
$$

where $L_{A}$ and $L_{B}$ are the length of the vehicles $\mathrm{A}$ and $\mathrm{B}$ respectively.

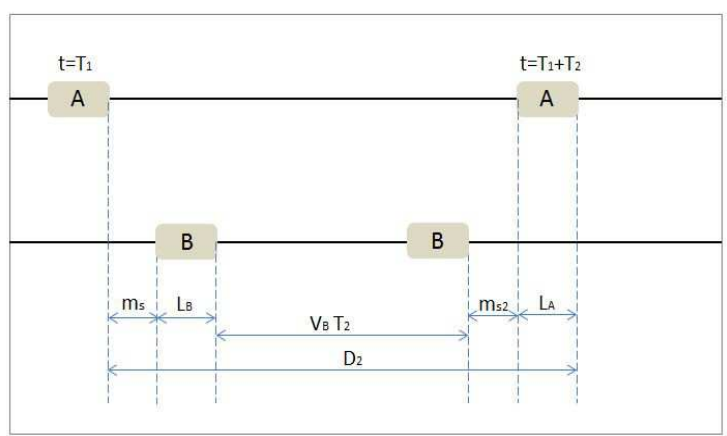

Fig. 3. Phase 2: Navigating on the left lane.

\section{Phase 3: Returning to the right lane}

In this phase, we make use of the symmetrical of the polynomials used in phase 1 . This leads to the same constraints that concern the dynamic stability of the vehicle ((5) and (6)). To guarantee the safety conditions, a third constraint is added claiming that the vehicle will reach the right lane while assuring a distance with the vehicle B greater than the security distance (the security distance is taken as 2 seconds dealing with the road rules [8]). Then, referring to Fig. 4, we have to guarantee the following :

$$
D_{o b s_{2}}=D_{3}-V_{B} T_{3}+m_{s 2} \geq 2 V_{B},
$$

where $D_{o b s_{2}}$ is the distance between the vehicles A and B on the right lane once the returning phase is executed, $D_{3}$ and $T_{3}$ are the traveling distance and time of this phase. Now, let $V_{A_{i 2}}$ and $V_{A_{f 2}}$ be the initial and the target speed of the vehicle A for this phase. $D_{3}$ is then computed by $\left(V_{A_{i 2}}+V_{A_{f 2}}\right) T_{3} / 2$. Eq. (14) is then written as:

$$
V_{A_{f 2}} \geq 2 / T_{3}\left(2 V_{B}-m_{s 2}+V_{B} T_{3}-V_{A_{i 2}} T_{3} / 2\right)
$$

The dynamic constraints for this phase are similar to those of phase 1 and are given by:

$$
\begin{aligned}
V_{A_{f 2}} & \leq \min \left(V_{A_{i 2}}+2 / 3 a_{x_{\max }} T_{3}, V_{x_{\max }}^{L_{1}}\right) \\
T_{3} & \geq \max \left(\sqrt{\frac{5.77 w}{a_{y_{\max }}}}, \sqrt{\frac{-5.77 w}{a_{y_{\min }}}}\right) .
\end{aligned}
$$

In order to find $T_{3}$ and $V_{A_{f 2}}$ that satisfy (15), (16) and (17), we have to guarantee that the solution space for equations (15) and (16) is not empty. After some computations, we can find that this solution space is not empty if and only if we take $T_{3}$ in such a way to satisfy the following conditions:

$$
\begin{gathered}
T_{3} \geq \frac{-V_{A_{i 2}}+V_{B}+\sqrt{\left(V_{A_{i 2}}-V_{B}\right)^{2}+8 / 3 V_{B} a_{x_{\max }}}}{2 / 3 a_{x_{\max }}} \\
T_{3} \geq \frac{2\left(m_{s 2}-2 V_{B}\right)}{2 V_{B}-V_{A_{i 2}}-V_{x_{\max }}^{L_{1}}}
\end{gathered}
$$

From (19), (18) and (17), We can define $T_{3}$ as :

$$
T_{3} \geq \max \left(T_{\max 1}, T_{\max 2}, T_{\max 3}\right),
$$

with

$$
\begin{aligned}
& T_{\max 1}=\max \left(\sqrt{\frac{5.77 w}{a_{y_{\max }}}}, \sqrt{\frac{-5.77 w}{a_{y_{\min }}}}\right) \\
& T_{\max 2}=\frac{-V_{A_{i 2}}+V_{B}+\sqrt{\left(V_{A_{i 2}}-V_{B}\right)^{2}+8 / 3 V_{B} a_{x_{\max }}}}{2 / 3 a_{x_{\max }}} \\
& T_{\max 3}=\frac{2\left(m_{s 2}-2 V_{B}\right)}{2 V_{B}-V_{A_{i 2}}-V_{x_{\max }}(\text { Lane } 1)} .
\end{aligned}
$$

Once $T_{3}$ is defined by (20), $V_{A_{f 2}}$ is set by choosing any value satifying (15) and (16).

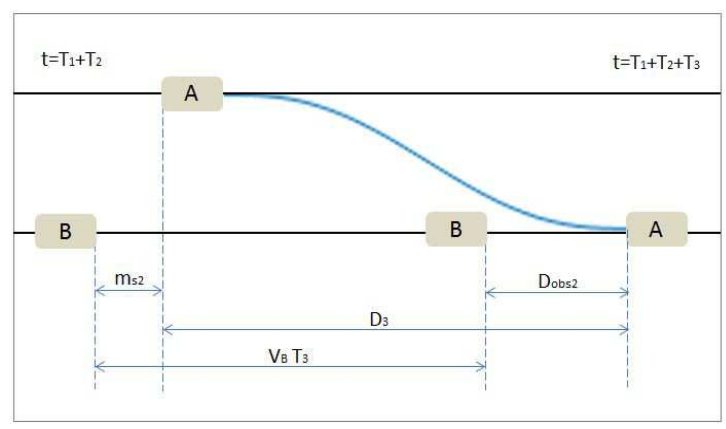

Fig. 4. Phase 3: Returning to the right lane.

\section{Local Path Planning using Clothod Tentacles METHOD}

The local planning algorithm is executed every $100 \mathrm{~ms}$. A set of 41 tentacles is generated in the ego-centered reference frame related to the vehicle, at each step (see Fig. 5). Then, to guarantee a secure navigation, we generate around each tentacle a classification zone within a radius $d_{c}$ which is taken slightly greater than the vehicle width. In parallel, using sensors data, we construct an occupancy grid that represents the environment around the vehicle by a set of square cells $(800 * 800$ cells $)$ where each cell $(25 \mathrm{~cm} * 25 \mathrm{~cm})$ could correspond to a free or an occupied space. The superposition of the classification area with the occupancy grid allows to classify the tentacles between navigable and non navigable ones. In our approach, the tentacle is classified as non-navigable if an obstacle is detected within a distance of security $L_{c}$ 
corresponding to 2 seconds of reaction with respect to the ego vehicle speed. If the obstacle is beyond $L_{c}$, the tentacle is classified as navigable. Only one tentacle is chosen among the navigable tentacles using two criteria: The tentacle clearance $V_{\text {clearance }}$ and the tentacle convergence toward the reference trajectory $V_{\text {trajectory }}$. The first criterion expresses the distance that the vehicle can drive along a tentacle before hitting an obstacle, while the second defines the tentacle's convergence to a given reference trajectory. The best tentacle is then the tentacle that minimizes $V_{\text {clearance }}$ and $V_{\text {trajectory }}$ with a given compromise. If any navigable tentacle can be found, we proceed to brake the vehicle with a constant deceleration along the tentacle having the longest distance to the obstacle. For more details, please refer to [1].

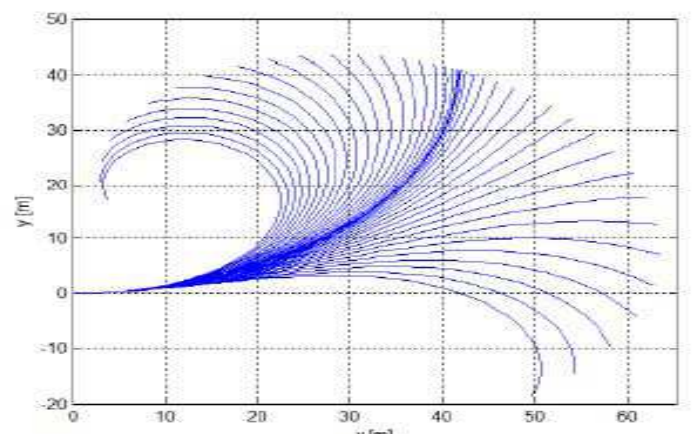

Fig. 5. A set of clothoids generated at a speed of $11 \mathrm{~m} / \mathrm{s}$ and a steering angle of $0.1 \mathrm{rad}$.

\section{Simulation Results}

To validate the developed navigation strategy, a simulation under Simulink/Matlab was conducted. We assume that the road is straight and the left lane is free so that the vehicle can overtake. The parameters $w, m_{s}, m_{s 2}$, and, the speed and the acceleration limits are set to:

$$
\begin{aligned}
& w=3.5 m, m_{s}=m_{s 2}=3 m \\
& a_{x_{\min }}=-2 \mathrm{~m} / \mathrm{s}^{2}, a_{x_{\max }}=1.5 \mathrm{~m} / \mathrm{s}^{2} \\
& a_{y_{\min }}=-4 \mathrm{~m} / \mathrm{s}^{2}, a_{y_{\max }}=4 \mathrm{~m} / \mathrm{s}^{2} \\
& V_{x_{\max }}^{L_{1}}=20 \mathrm{~m} / \mathrm{s}, V_{x_{\max }}^{L_{2}}=25 \mathrm{~m} / \mathrm{s}
\end{aligned}
$$

Many scenarios were tested, with different speeds for the ego and the overtaken vehicles. In the scenario shown in this section, the ego vehicle is navigating initially with $10 \mathrm{~m} / \mathrm{s}$ and the obstacle is navigating with $4 \mathrm{~m} / \mathrm{s}$. While navigating on the right lane, the ego vehicle detects the other vehicle and automatically adapts its speed in order to guarantee a secure inter-vehicle distance. Keeping in the mind that the desired speed of the ego vehicle is always $10 \mathrm{~m} / \mathrm{s}$ which is greater than $4 \mathrm{~m} / \mathrm{s}$ by more than $20 \mathrm{~km} / \mathrm{h}$ (equivalent to $5.55 \mathrm{~m} / \mathrm{s}$ ), and assuming that the left lane is free, the ego vehicle decides to overtake. A trajectory is then generated, as described in section III, where: $T_{1}=15.9583 \mathrm{~s}, V_{A_{i}}=10 \mathrm{~m} / \mathrm{s}, V_{A_{f}}=10 \mathrm{~m} / \mathrm{s}$ and $D_{1}=159 \mathrm{~m}$. The generated polynomial is shown in Fig. 6 and 7. As the desired vehicle speed is initially greater than
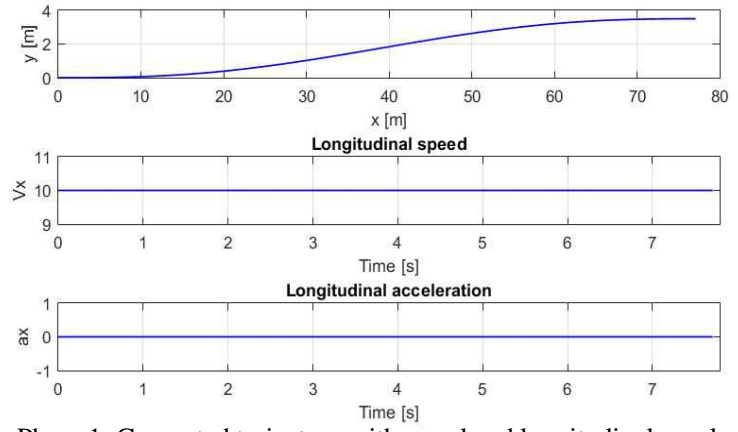

Fig. 6. Phase 1: Generated trajectory with speed and longitudinal accelerations profiles.
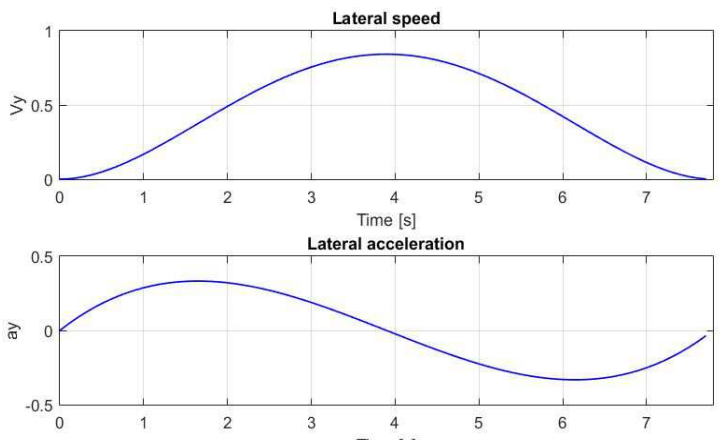

Fig. 7. Phase 1: Lateral speed ${ }^{\text {Time }}$ and acceleration profiles.

the overtaken vehicle speed, the lane changing is executed at constant speed.

The vehicle reaches the left lane at a position $\mathrm{p}$, when the vehicle $\mathrm{B}$ is at the position $p+m s$ on the right lane (see Fig. 8).

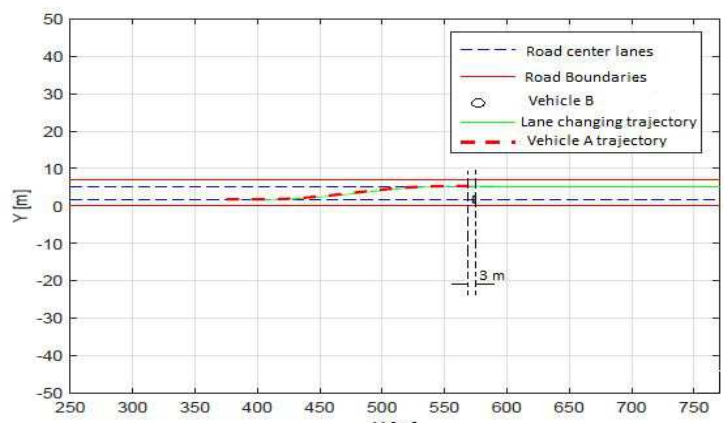

Fig. 8. Phase 1: Vehicle $\mathrm{A}^{\mathrm{X} \text { and } \mathrm{B}}$ positions at $t=T_{1}$.

The ego vehicle navigates on the left lane until it can return to the right lane. Once the ego vehicle is in front of the vehicle $\mathrm{B}$, the corresponding trajectory is generated, where $T_{3}=2.2 \mathrm{~s}$, $V_{A_{i 2}}=10 \mathrm{~m} / \mathrm{s}, V_{A_{f 2}}=12.24 \mathrm{~m} / \mathrm{s}$ and $D_{3}=24.42 \mathrm{~m}$. The generated trajectory for phase 3 is shown in Fig. 9 and 10. When the phase 3 is finished, the distance between the vehicles $\mathrm{A}$ and $\mathrm{B}$ is greater than $2 V_{B}=10 \mathrm{~m}$ (see Fig. 11). 


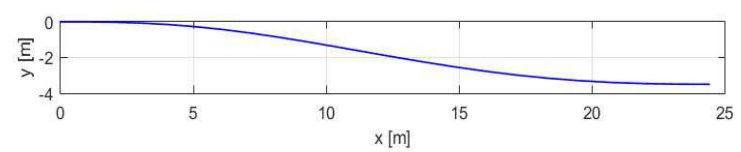

Longitudinal speed
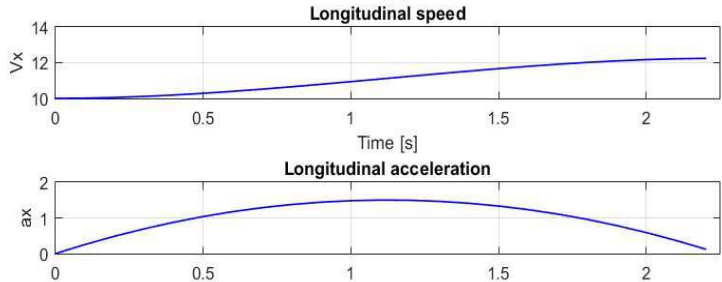

Fig. 9. Phase 3: Generated trajectory with speed and longitudinal accelerations profiles.
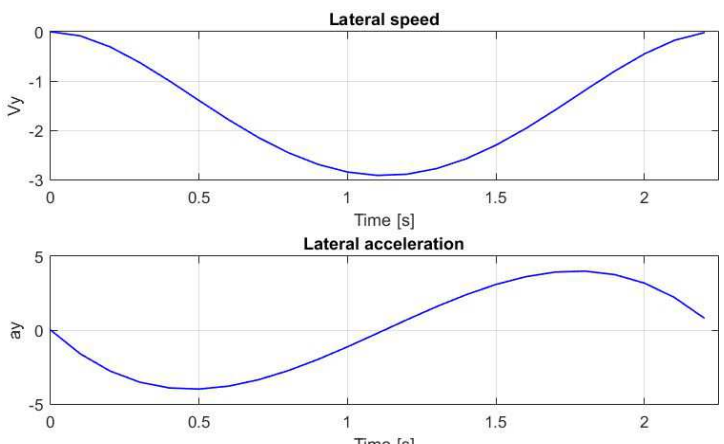

Fig. 10. Phase 3: Lateral sime [ș] and acceleration profiles.

\section{CONCLUSION}

In this paper, the trajectory planning for autonomous vehicles problem is addressed. Our domain of interest is essentially the overtaking maneuver associated with a local planning level. A strategy that satisfies the vehicle dynamics constraints as well as the road rules and the security measurements is presented to execute the overtaking maneuver. Indeed, this maneuver can be divided into three main maneuvers: Lane changing from right to left lane, navigating on the left lane and turning back from left to right lane. The corresponding trajectories for the lane changing phases are generated in such a way to guarantee the vehicle stability and security while respecting the road rules. At the local level, the tentacles method with clothoids form is used in order to guide the vehicle to track the generated trajectories while avoiding any possible collision. The algorithm was validated under Simulink/Matlab, considering low speeds for both the ego and the overtaken vehicle. For future works, we aim to consider high speeds scenarios since the objective is to plan trajectories on highways. Another important point to be considered is the representation of curved roads since the vehicle stability is more threatened on a curved road.

\section{ACKNOWLEDGMENT}

This work was carried out in the framework of the Labex MS2T, which was funded by the French Government, through the program Investments for the future managed by the $\mathrm{Na}-$ tional Agency for Research (Reference ANR-11-IDEX-0004-

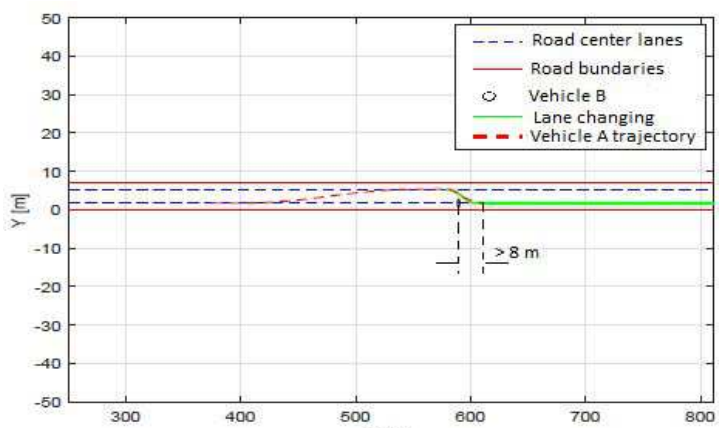

Fig. 11. Phase 3: Vehicle $\mathrm{A}^{[\mathrm{m}]}$ and $\mathrm{B}$ positions at $t=T_{3}$.

02) and the framework of the Equipex ROBOTEX (Reference ANR-10-EQPX-44-01), which was funded by the French Government, through the program Investments for the future managed by the National Agency for Research.

\section{REFERENCES}

[1] A. Chebly, G. Tagne, R. Talj and A. Charara, "Local trajectory planning and tracking of autonomous vehicles, using clothoid tentacles method," IEEE Intelligent Vehicles Symposium (IV), Seoul, Korea, 2015.

[2] T. Shamir, "How should an autonomous vehicle overtake a slower moving vehicle: Design and analysis of an optimal trajectory," IEEE Transactions on Automatic Control, vol. 49, pp. 607-610, 2004.

[3] J. Nilsson, M. Brännström, E. Coelingh and J. Fredriksson, ’Longitudinal and lateral control for automated lane change maneuvers," American Control Conference (ACC), 2015.

[4] I. Papadimitriou and M. Tomizuka, "Fast lane changing computations using polynomials," American Control Conference, vol. 1, pp. 48-53, 2003.

[5] V. Duggal, K. Bipin, A.K. Singh, B. Gopalakrishnan, B.K. Bharti, A. Khiat and K. Krishna, "Overtaking maneuvers by non linear time scaling over reduced set of learned motion primitives," Intelligent Vehicles Symposium (IV), pp.115-120, 2015.

[6] R. Schubert, K. Schulze and G. Wanielik, "Situation assesment for automatic lane-change maneuvers," IEEE Transactions on Intelligent Transportation Systems, vol.11, pp.607-616, 2010.

[7] J. Naranjo, C. Gonzalez, R. Garcia and T. De Pedro, "Lane-change fuzzy control in autonomous vehicles for the overtaking maneuver," IEEE Transactions on Intelligent Transportation Systems, vol.9, pp.438-450, 2008.

[8] J. Bauer, "Rules of the road," 2005.

[9] S. Sundar and Z. Shiller, "Emergency lane-change maneuvers of autonomous vehicles," Journal of Vehicle Dynamic Systems, Measurement, and Control, vol.120, pp.37-44, 1998

[10] W. Chee and M. Tomizuka, "Lane change maneuver for AHS applications,” JSAE Review, vol.2, pp.219, 1995.

[11] L. Ching-Lu, J. Jyh-Ching and C. Shun-Hung, "Robust lane-changing maneuver," 2014

[12] S. Glaser, B. Vanholme, S. Mammar, D. Gruyer and L. Nouveliere, "Maneuver-based trajectory planning for highly autonomous vehicles on real road with traffic and driver interaction," IEEE Transactions on Intelligent Transportation Systems, vol.11, pp.589-606, 2010.

[13] J. Nilsson, M. Brnnstrm, E. Coelingh and J. Fredriksson, "Lane Change Maneuvers for Automated Vehicles," IEEE TRANSACTIONS ON INTELLIGENT TRANSPORTATION SYSTEMS, 2016 